\title{
DISSS/ET Modernization Feasibility of Adapting Existing Software and Hardware Technologies
}

R. Scott Strait and Fission Energy and System Safety Program

September 30, 1993

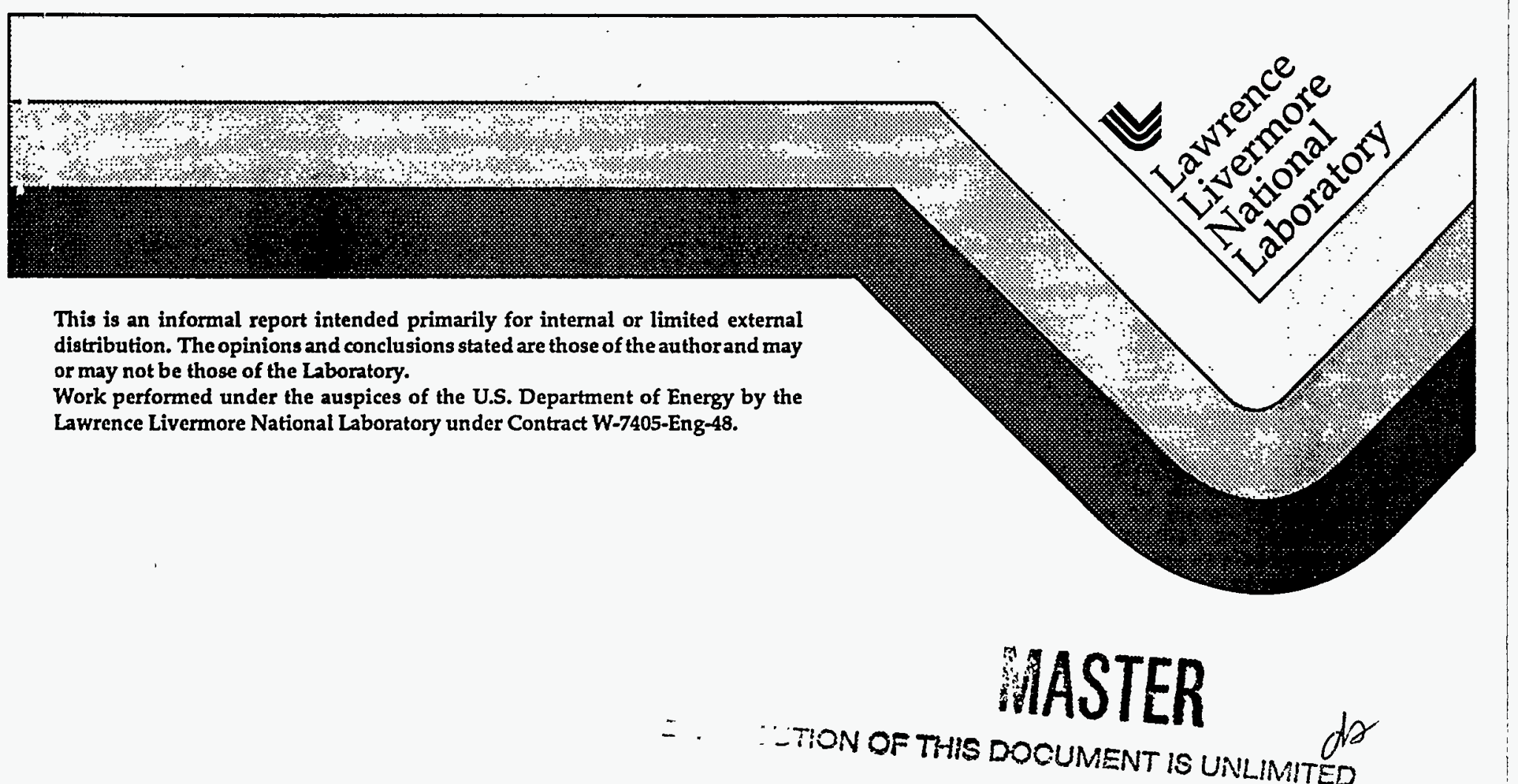




\section{DISCLAIMER}

This document was prepared as an account of work sponsored by an agency of the United States Government. Neither the United States Government nor the University of California nor any of their employees, makes any warranty, express or implied, or assumes any legal liability or responsibility for the accuracy, completeness, or usefulness of any information, apparatus, product, or process disclosed, or represents that its use would not infringe privately owned rights. Reference herein to any specific commercial product, process, or service by trade name, trademark, manufacturer, or otherwise, does not necessarily constitute or imply its endorsement, recommendation, or favoring by the United States Government or the University of California. The views and opinions of authors expressed herein do

- not necessarily state or reflect those of the United States Government or the University of California, and shall not be used for advertising or product endorsement purposes.

This report has been reproduced directly from the best available copy.

Available to DOE and DOE contractors from the

Office of Scientific and Technical Information

P.O. Box 62, Oak Ridge, TN 37831

Prices available from (615) 576-8401, FTS 626-8401

Available to the public from the

National Technical Information Service

U.S. Department of Commerce

5285 Port Royal Rd.,

Springfield, VA 22161

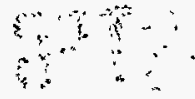




\section{Table of Contents}

Section 1

Introduction .... Page 1

Section 2

LLNL EC/EDI Approach \& Software .... Page 3

2.1 Transport Source Data to Clearance Office .... Page 3

2.2 Review/Store Source Data at Clearance Office .... Page 3

2.3 Transport QSP Packet to DOE Field Office .... Page 4

2.4 Review/Store QSP Packet at DOE Field Office .... Page 4

2.5 Transport QSP Packet to OPM .... Page 4

2.6 Update PIPS database at OPM .... Page 4

2.7 Transport Investigation Report to DOE Field Office .... Page 4

2.8 Review/Store Investigation Report at DOE Field Office .... Page 4

2.9. Review Investigation Report/Make Clearance Decision .... Page 5

2.10 Transport Clearance decision to Contractor Site .... Page 5

2.11 Review/Store Data at Clearance Office .... Page 5

2.12 Issue Approved Clearance/Badge to Applicant .... Page 5

Section 3

SF-86 Software Applications .... Page 6

3.1 User Interface Requirements .... Page 6

3.2 PerFORM PRO Software .... Page 7

3.3 Features Comparison of Leading Forms Packages .... Page 8

Section 4

Electronic Fingerprint Systems .... Page 11

4.1 Extracting Fingerprint Image Data .... Page 11

4.2 FBI-approved Fingerprint Systems .... Page 12

4.3 How Information was gathered .... Page 12 
4.4 DBI's Tenprinter System and Remote Server (Delta Box) .... Page 12

4.5 Indentix's Touchprint System .... Page 13

4.6 Comparative Analysis Chart .... Page 14

4.7 Another Possibility - PC Gateway Concept .... Page 15

4.8 Recommendation .... Page 15 


\section{SECTION 1}

\section{Introduction}

The Fission Energy and Systems Safety Program (FESSP) at LLNL was funded by the DOE Office of Safeguards and Security to develop an integrated system, hereafter referred to as the Security Clearance Electronic Processing, Transfer, Evaluation, and Recordkeeping (SCEPTER) System, for the electronic collection and transfer of personnel security data between clearance offices at contractor sites and DOE field offices and the Office of Personnel Management (OPM). This system, will use existing software and hardware technologies where feasible.

The project will consist of three phases. The first phase will investigate user needs, determine the feasibility of using existing technologies, and define project requirements. The second phase will be a pilot project to develop the computer systems and procedures required to automate the security clearance work flow within DOE and between DOE and OPM. Once the pilot system is implemented and tested, the decision to extend the system throughout DOE can be made. This third phase, the extension to a full production system, will require the investment of considerable funds in equipment and in the development of both a computer system and management infrastructure to support its successful operation. This investment will be undertaken only after the pilot system is operational and evaluated.

As part of the first phase, we are performing a requirements analysis to determine users' needs. We conducted a survey at many of the DOE field offices and contractor sites using a user input form. These comments will be summarized and documented in the requirements analysis. We are visiting a number of DOE field offices, contractor sites and OPM to determine user requirements for the SCEPTER System. These user requirements will be the basis for developing the system design.

Also as part of the first phase, we have examined the feasibility of adapting the following existing software and hardware technologies for use in the electronic security clearance process:

- LLNL EC/EDI approach and software

- SF-86 software applications

- Electronic fingerprint systems

This report presents the results of our review. Based on this review, we are confident that all of these existing technologies and systems can be adapted and integrated to develop an electronic security clearance processing system for the DOE. 


\section{LLNL EC/EDI Approach \& Software}

The LLNL EC Project has developed an approach, Electronic Commerce Through Electronic Data Interchange (EC/EDI), using a blend of commercial resources and government infrastructure to implement electronic commerce on a large scale at low cost. The implementation of Electronic Commerce using this approach involves the integration of commercial software, LLNL-developed software, and hardware with local systems. While many of the pieces needed to implement the LLNL EC/EDI Approach are available off-the-shelf, the integration of these pieces requires some custom tailoring to meet the specific needs of the DOE.

We have investigated the feasibility of using the LLNL EC/EDI approach and software in an electronic security clearance processing system. Trusted multipart electronic mail messages are an efficient and secure means for transporting security clearance data between contractor site clearance offices and DOE field offices, and between DOE field offices and OPM. Many of the EC/EDI software technologies could provide effective components of an electronic clearance processing system. We have conducted a preliminary review of the security clearance process and have briefly described how the EC/EDI approach and software can be adapted to perform each task in the electronic process.

\subsection{Transport Source Data to Clearance Office}

The data collected (see Section 3) and the fingerprints (see Section 4) will be placed in a trusted multi-part electronic mail message and moved to the clearance office using the following electronic mail systems:

- Distributed Electronic Mail (DEM) developed by the LLNL EC project for the DOS PC.

- Techmail for the Macintosh.

- Trusted Electronic Mail (EM) developed by the LLNL EC project for UNIX platforms.

\subsection{Review/Store Source Data at Clearance Office}

The data in the multi-part trusted electronic mail message will be extracted into a database on the clearance office Site Gateway using the Oracle RDBMS (Relational Data Base Management System). A SCEPTER database application will be written to store, display, update and retrieve information to the Oracle database. The LLNL EC/EDI Distributed User Interface (DUI) will allow users to review and edit information in the database. 


\subsection{Transport QSP Packet to DOE Field Office}

The data in the trusted multi-part electronic mail message will be moved from the clearance office Site Gateway to the DOE field office Site Gateway using LLNL EC/EDI Electronic Mail.

\subsection{Review/Store QSP Packet at DOE Field Office}

The data in the trusted multi-part electronic mail message will be extracted into a database on the DOE field office Site Gateway using the Oracle RDBMS. The SCEPTER database application will be used to store, display, update and retrieve information to the Oracle database. The LLNL EC/EDI DUI will allow users to review and edit information in the database.

\subsection{Transport QSP Packet to OPM}

The required data in the trusted multi-part electronic mail message will be moved from the DOE field office Site Gateway to the OPM Site Gateway using LLNL EC/EDI Electronic Mail.

\subsection{Update PIPS database at OPM}

The PIPS database will be updated with the DOE field office information from the OPM Site Gateway using a batch-file transfer method.

\subsection{Transport Investigation Report to DOE Field Office.}

Investigation reports will be retrieved and transferred from the PIPS database to the OPM Site Gateway using a batch-file transfer method. The reports will be placed in a trusted electronic mail message and sent to the DOE field office Site Gateway using LLNL EC/EDI Electronic Mail.

\subsection{Review/Store Investigation Report at DOE Field Office}

The investigation report data in the trusted electronic mail message will be extracted into a database on the DOE field office Site Gateway using the Oracle RDBMS. The SCEPTER database application will be used to store, display, update and retrieve information to the Oracle database. The LLNL EC/EDI DUI will allow users to review and edit information in the database. 


\subsection{Review Investigation Report/Make Clearance Decision}

The DOE field office will review the investigation report and make a clearance decision. The clearance decision data will be placed in the database using the QSP database application. The LLNL EC/EDI DUI will allow users to review and edit information.

\subsection{Transport Clearance decision to Contractor Site}

The DOE field office clearance decision data will be extracted from the database into a trusted electronic mail message and sent to the clearance office Site Gateway using LLNL EC/EDI Electronic Mail.

\subsection{Review/Store Data at Clearance Office}

The clearance decision data in the trusted electronic mail message will be extracted into the database on the clearance office Site Gateway using the Oracle RDBMS. The SCEPTER database application will be used to store, display, update and retrieve information to the Oracle database. The LLNL EC/EDI DUI will allow users to review and edit information in the database.

\subsection{Issue Approved Clearance/Badge to Applicant}

The appropriate clearance and badge will be issued to the applicant and the Oracle database on the clearance office Site Gateway will be updated using the SCEPTER database application and the LLNL EC/ED DUI software. 


\section{SF-86 Software Applications}

LLNL has completed a preliminary analysis of the HQ DOE-funded SF-86 software using PerFORM Pro software. The basic features of the software are:

- Is MS DOS-based, but does not require Windows or a "fast" PC.

- Uses the Perform Pro software application.

- Requires an additional piece of commercial software to perform printing and data validation: Datamerge, from Delrina Corporation. This software is on order.

- Does not have any provision for use on a Macintosh or UNIX platform.

- Does some data validation, e.g., will not permit a date of 00/00/00.

- Stores its data in a series of dBase-compatible tables; written in the Clipper language.

- Requires an HP Laser Jet II or PCL3 compatible.

The primary function of this software is to produce valid printed forms. While we can report that it has some validation features, as described above, we are awaiting the arrival of the required additional software to test the complete data validation and printing capabilities.

Our review indicates that it is feasible and prudent to adapt and expand the capabilities of the HQ DOE-funded SF-86 software by using PerFORM Pro Designer under Windows and PerFORM Pro Filler under Windows, DOS and Macintosh. It is also feasible to extract data from these forms packages into a trusted electronic mail message for transport to the clearance office Site Gateway.

\subsection{User Interface Requirements}

One of the major factors in the design of a system are the internal user interfaces available to users for collecting source data. Many of the comments and requirements received from the User Input Form survey and the local clearance office were directly related to the source data collection process. A summary of these comments include:

- user-friendly interface for QSP applicants

- multiple methods of data collection

- intelligent forms package with sophisticated data validation capabilities

- PC and Macintosh support

- data validation of dates and cross-referencing between dates of domicile, education and employment 
The product used in the creation of the HQ DOE-funded SF-86 application was a combination of PerFORM PRO and the Clipper ${ }^{\text {TM }}$ database language. Preliminary analysis of product reviews indicates that PerFORM PRO is the leading product in this area. However, the result delivered does not take advantage of the user interface capabilities of Windows and PerFORM PRO. Among other features, PerFORM PRO permits the design of a form in Windows that can be used in all three personal computer platforms:

- Windows

- MS DOS text mode

- Macintosh

We are also anaiyzing the user interface and data validation features of other SF-86 software developed for: INEL, which also uses PerFORM PRO; WHC, which uses Jet Form; and SRS.

\subsection{PerFORM PRO Software}

PerFORM PRO Designer and Filler allow for designing, filling-in and maintaining customized electronic business forms. PerFORM PRO allows program developers and users to:

- Create multiple page forms

- Form design locking for added security

- Link data between forms and organize form "folders" for easy access

- Write forms data directly to dBASE and ASCII database formats

- Search on forms data stored in dBASE format

- Modify forms without losing data - data structure maintained if stored in dBASE format

- Database "look up and replace" with dBASE files or data from other forms

- dBASE field references, automatic index creation and maintenance, memo fields

- Graphics file support - TIFF, GEM Image, MacPaint, PC Paintbrush, GEM Line Art, EPSF, Windows metafile and compatible formats

- Automatic fill in of fields, including date and time, serial numbers, page numbers, checked marked boxes, default entries

- Automatic calculations with mathematical, logical, financial and other functions

- User defined functions

- Fast mode for speedy form filling

- Data entry validation, including numeric checks, ranges, tables, compulsory fields 
- Online filler help with customized functions

- Enhanced printing speed and features

- Support for macros, cartridges and downloadable fonts.

\subsection{Features Comparison of Leading Forms Packages}

We recommend using PerFORM PRO over JetForm Designer because the existing SF-86 software already uses PerFORM PRO and PerFORM PRO offers stronger forms design, data management, and programmability.

In the August 1993 edition of PC World, in the article entitled "Electronic Forms Fills the Bill", by Scott Spanbauer, an evaluation of the four top Windows forms processing packages was provided. The outcome of the article was that the two leading contenders (referred to as the Best Buys) are PerFORM PRO and Jet Form. The article stated that "either program is an excellent choice for a forms processing application, but be sure to consider which one's strengths most suits your needs". The article also stated that PerFORM PRO is "the most sophisticated forms processing package" and that its contender, JetForm does not offer as many design and data management options as PerFORM PRO. Most notably, JetForm does not incorporate a fully relational database.

The following information was provided in a feature comparison table on the following pages: 


\begin{tabular}{|c|c|c|}
\hline FEATURES & 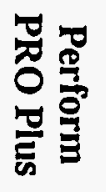 & 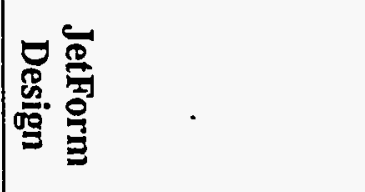 \\
\hline \multicolumn{3}{|l|}{ Standard Features } \\
\hline List Price & $\$ 399$ & $\$ 495$ \\
\hline Context-sensitive help & yes & yes \\
\hline Quick-start guide & yes & yes \\
\hline \multicolumn{3}{|l|}{ Forms Design } \\
\hline Sample forms includes & 150 & 12 \\
\hline Multi-page forms & yes & yes \\
\hline Multipart forms & no & yes \\
\hline Imports TIFF and PCX formats & yes & Black and white only \\
\hline Aligns objects & yes & yes \\
\hline Groups objects & yes & yes \\
\hline Traces scanned forms & yes & yes \\
\hline \multicolumn{3}{|l|}{ Data Handling } \\
\hline One-to-many relationships & yes & no \\
\hline Calculated fields & yes & yes \\
\hline Info linked among forms & yes & yes \\
\hline Field masking & yes & yes \\
\hline Compulsory fields & yes & yes \\
\hline Look-up tables & yes & yes \\
\hline Specify field fill-in order & yes & yes \\
\hline $\begin{array}{l}\text { Generates serial numbers automati- } \\
\text { cally }\end{array}$ & yes & no \\
\hline \multicolumn{3}{|l|}{ Data File Formats Read/Written } \\
\hline DBF & yes & yes \\
\hline NDX & yes & no \\
\hline Paradox & yes & yes \\
\hline ASCII & yes & yes. \\
\hline OLE/DDE support & yes & yes \\
\hline \multicolumn{3}{|l|}{ Forms Management } \\
\hline Long file descriptions & yes & yes \\
\hline Forms linking & yes & yes \\
\hline
\end{tabular}




\begin{tabular}{|c|c|c|}
\hline FEATURES & 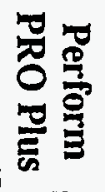 & 施总 \\
\hline Forms locking & yes & yes \\
\hline Hides field data & yes & yes \\
\hline Read-only fields & yes & yes \\
\hline Password protection & yes & no \\
\hline \multicolumn{3}{|l|}{ Printing } \\
\hline $\begin{array}{l}\text { Supports downloaded and cartridge } \\
\text { fonts }\end{array}$ & yes & yes \\
\hline Downloads multipage formats & yes & yes \\
\hline \multicolumn{3}{|l|}{ Printer Support } \\
\hline HP LaserJet II/III & yes & yes \\
\hline PostScript & yes & yes \\
\hline Epson LQ dot matrix & yes & data only \\
\hline HP DeskJET & yes & $\begin{array}{l}\text { uses Windows printer } \\
\text { driver }\end{array}$ \\
\hline
\end{tabular}


SECTION 4

\section{Electronic Fingerprint Systems}

The live-scan workstations are FBI-approved, commercially provided fingerprint system that produces forensic-quality tenprint records by electronically scanning and capturing the live fingerprints of a subject.

Typically, it is a turn-key system comprising of a PC, scanner, laser printer, graphics display, and communication equipment integrated into a custom cabinet with a software front-end.

The cited advantages of these systems over traditional inked method are:

- Better quality tenprints.

- Multiple copies of tenprints can be generated from a single scan.

- Fingerprints may be transmitted to remote printer or uploaded to the FBI mainframe-based Automatic Finger Identification System (AFIS).

Our review indicates that it is practical to access, extract, store, and forward live-scan fingerprint images using a vendor-supplied Remote Server. We are also pursuing the feasibility of a less expensive and more flexible approach to access and extract the fingerprint images. The images can be transported using electronic mail, and then submitted to AFIS or sent to a printer. Images are not interchangeable between the companies' products.

\subsection{Extracting Fingerprint Image Data}

These workstations are typically non-intrusive, closed systems, and geared towards the hardcopy environment. Exporting captured graphics images to the outside world was not the developers' design concerns, and consequently, images are handled and kept in a non-standard, proprietary way.

Our objective is to extract fingerprint images from the live-scan workstations, and move it electronically along with QSP data. Due to the closed nature of the systems, extracting or importing fingerprint images directly from these machines is non-trivial, and requires fairly extensive changes to the existing software, which the vendors are reluctant to undertake. If the workstation could act as a node of a LAN sharing its hard disk with other nodes, then we would be able to easily get to the image files. Unfortunately, the proprietary database is not that easily accessible. In addition, another unknown variable is whether the workstation can act as a node of a LAN without causing conflicts with the existing software. The vendors' technical staff were skeptical about this method.

The good news is that all the vendors provide a so-called store-and-forward remote server whose primary function is to route the images to a remote printer 
or to the FBI mainframe-based Automated Fingerprint Identification System (AFIS). These remote servers are based on open architecture, and can be serendipitously utilized by us to export and import the live-scan images to the outside world almost in a seamless way.

\subsection{FBI-approved Fingerprint Systems}

The FBI Identification Division (ID) announced in its memo dated November 25,1991 , the list of vendors whose workstations meet the FBI standard. There were only three in the list. Margie Hall of $\mathrm{ID}$ (phone 202-324-5257) verified that the list has not changed. The vendors are: Digital Biometrics, Inc. (DBD); Identix, Inc.; and Fingermatrix, Inc.

Fingermatrix, Inc. shows signs of troubled company. Phone calls are rarely answered or returned. If calls are answered, it is with an answering service who is unable to give out any technical information. When contact was finally made with Mr. Raymond Norat of Fingermatrix, he politely asked that all inquiries be submitted by fax, and assured a one-day turnaround time for the reply. After one week and several more futile attempts to reach him again, the two pages of questions are yet to be answered. Because no literature is available from Fingermatrix, discussions are limited to the other two main players. Both are reputable companies and manufacture solid products. We will continue to attempt to learn more about the Fingermatrix system.

\subsection{How Information was gathered}

This paper is based on field visits to observe the units in real operation and to inspect the physical hardware unit and software modules, meetings and phone calls with vendor $R \& D$ personnel, and vendor product literature.

\subsection{DBI's Tenprinter System and Remote Server (Delta Box)}

DBI's Tenprinter (currently in use at the LLNL clearance office) has a larger installed base than its competitor, the Identix TouchPrint system. Enclosed in a custom-built metal cabinet, the unit seems to operate solidly. According to the staff, they have scanned over a thousand tenprints with very little problem. Powered by a 486-based PC and running under DOS with Desquiew, the Tenprinter drives a scanner, graphics display terminal, and a custom-enhanced HP LaserJet III laser printer. The image downloaded to the LaserJet is not in HPCL format, and needs to be translated by the on-board chip set in the enhancement unit. 
The software user-interface is easy-to-use and user-friendly. It is a turn-key system that does not allow anyone into the operating system layer. Scanned images are inaccessible from DOS and kept in a proprietary format in the graphics unit. Thus, it is pointless to try to get to it from DOS level. Only the last twenty images are kept online due to space limitations.

The Remote Server (Model 2102R, dubbed Delta Box) is a UNIX box with a Motorola processor. It can be connected to the Tenprinter via an asynchronous serial line, Ethernet, or T1. The Remote Server appears on Tenprinter as one of its print destinations. When a scanned image is sent to the Remote Server, it is captured and stored under a UNIX directory. The individual image gets stored under a distinct subdirectory along with the biographic information of the subject. The good news is that the Remote Server supports ftp, meaning that these images can be freely extracted. The vendor was unsure if it also supports NFS (Network File System). In any event, one DBI employee says he regularly logs into these Remote Servers and performs system administration at the UNIX prompt level.

The Remote Server can forward images to another Remote Server, print hardcopy on local or remote laser printers, or directly upload images to the Printrak AFIS. At the moment, the Remote Server does not support the interface to NEC AFIS.

The Remote Server can also receive finger-scan images from any UNIX box an important consideration for our project - because the FBI will ultimately need that capability to process fingerprints electronically. We would need the DBI "Bridge" product for the capability to upload to the Remote Server from other machines to their machine.

\subsection{Indentix's Touchprint System}

The Unit seemed to operate flawlessly, and its cabinet and hardware units are aesthetically more pleasing than the Tenprinter. In terms of software and hardware architecture, it resembled DBI's Tenprinter in all aspects. Accessing or extracting fingerprint images directly from the TouchPrint system is very difficult and is discouraged by the vendor. Instead, they recommend the use of their remote server.

Identix has just announced a product named the Store-and-Forward Server. It is IBM PS/2 based machine running under OS/2. It can be connected to the TouchPrint using the IBM LAN Server over Ethernet or token ring, or through a serial dial-up. It can route the image files to another Store-and-Forward box, a local printer, or a remote printer controller. The literature does not mention any interface to AFIS. 
The Identix engineer stated that they have successfully installed NFS (vendor: Beamann \& White) on TouchPrint, thus making their image files accessible to other client machines. Industry's most popular PC-NFS by SunSelect does not run, he says, due to available memory constraint.

The ability to run NFS on a workstation offers a less expensive solution than purchasing the vendor's remote server.

\subsection{Comparative Analysis Chart}

\begin{tabular}{|c|c|c|}
\hline & Digital Biometrics, Inc. & Identix, Inc. \\
\hline Product Name: & Tenprinter & TouchPrint \\
\hline Processor: & 486-PC & IBM PS 2,80386 based \\
\hline Operating System: & DOS with Desquiew & DOS with Desqview \\
\hline RAM: & Unspecified & $2 \mathrm{MB}$ \\
\hline Hard Disk & $\begin{array}{l}300 \mathrm{MB} \text { standard } \\
\text { higher available }\end{array}$ & $\begin{array}{l}80 \mathrm{MB} \text { standard } \\
\text { higher available }\end{array}$ \\
\hline Maximum Scan Resolution: & $400 \times 300 \mathrm{dpi}$ & $500 \times 500 \mathrm{dpi}$ \\
\hline Laser Printer: & $\begin{array}{l}\text { HP LaserJet III with proprietary } \\
\text { modification }\end{array}$ & $\begin{array}{l}\text { HP LaserJet III with proprietary } \\
\text { modification }\end{array}$ \\
\hline Printer Price: & Bundled & $\$ 5,700$ for single side. \\
\hline Can use generic laser printer: & NO & NO \\
\hline Max Images kept on-line: & $<25$ & $<25$ \\
\hline FBI Certification: & YES & YES \\
\hline Graphic Image Format: & Proprietary & TIFF \\
\hline Graphic Image Size: & $2.5 \mathrm{MB}$ & $\begin{array}{l}1.5 \mathrm{MB} \text { for } 300 \mathrm{dpi} \\
8 \mathrm{MB} \text { for } 500 \mathrm{dpi}\end{array}$ \\
\hline $\begin{array}{l}\text { Meets proposed NIST ANSI scanning } \\
\text { standard: }\end{array}$ & NO & YES \\
\hline Image Reject Check: & NO & YES \\
\hline Interface with AFIS: & YES & YES \\
\hline Can Remote Print: & YES & YES \\
\hline Remote Server: & YES & YES \\
\hline Remote Server Architecture: & UNIX Hosted & OS/2 based IBM/PS2 \\
\hline Remote Server Storage Capacity: & 1.860 tenprints & 125 tenprints \\
\hline File Accessibility: & Open & Needs user enhancements \\
\hline Purpose of Remote Server: & Store and Forward & Store and Forward \\
\hline
\end{tabular}




\begin{tabular}{|l|l|l|}
\hline & Digital Biometrics, Inc. & Identix, Inc. \\
\hline Company Address: & $\begin{array}{l}\text { 1250 N. Lakeview, Suite I. } \\
\text { Anaheim. CA 92807 }\end{array}$ & $\begin{array}{l}\text { 510 N. Pastoria Ave, } \\
\text { Sunnyvale. CA 94086 }\end{array}$ \\
\hline Phone: & $(714) 777-1278$ & $(408) 739-2000$ \\
\hline FAX: & $(714) 777-1908$ & $(408) 739-3308$ \\
\hline Point of Contact: & Dennis Labriola & Fred Mazzi \\
\hline System Price: & $\$ 44 \mathrm{~K}$ & $\$ 33 \mathrm{~K}$ \\
\hline Remote Server Cost: & $\$ 40 \mathrm{~K}$ & $\$ 21 \mathrm{~K}$ \\
\hline Maintenance Contract Cost: & $\$ 4,080 / \mathrm{yr} 24 \mathrm{hrs}$ a day & $\$ 1,740 / \mathrm{yr}$ for 8hrs a day \\
\hline
\end{tabular}

\subsection{Another Possibility - PC Gateway Concept}

So far, we have looked at methods to access the tenprint images on live-scan workstations using only vendor-provided equipment. There is an alternate way, which, if proven to work, may offer the least expensive and vendor-independent method.

The idea calls for inserting a PC between the live-scan workstation and the vendor-modified laser printer. Dubbed the PC Gateway, the PC can be programmed to mimic the laser printer and capture the images sent from the workstation and route them to a Site Gateway. The PC can conceivably offer additional services such as QSP form processing. In other words, it does not have to be a dedicated image capture box.

The laserprinter may be completely dispensed with if this scheme proves successful. One advantage of the PC Gateway is that it should work with any vendors' product.

The concept will not work, however, if the vendor designed the laser printer to perform a software handshake with the workstation. It would not be easy to emulate the handshake. Currently, a proof-of-concept test is under way.

\subsection{Recommendation}

In so far as workstations' capabilities are concerned, both seem capable. It follows, then, that the decision is based on the accessibility of the fingerprint images; which is directly related to the Remote Server's architecture and its capabilities. 
DBI's Remote Server is field-proven, and based on UNLX - the open system standard. Few problems are expected with the Remote Server's capabilities to interoperate with the Site Gateways that will be placed at contractor sites, DOE field offices and at OPM.

Identix's Store-and-Forward box is based on OS/2 - another good operating system. However, the system software is relatively new and untested. Also, it remains to be seen how well it would interoperate with the Site Gateways."

Identix's TouchPrint system, if it works well with NFS, may offer the most costeffective, vendor-assisted solution.

If proven to work, the PC Gateway concept may be the most appealing solution because it is the least expensive way, and does not preclude any vendor's product. If remote servers are required, we recommend DBI's Remote Server solution because of its relatively-easy interconnection with Site Gateways allowing the electronic transfer of fingerprint files. We are actively pursuing the PC Gateway idea because the benefits that it offers are worthwhile. 
